\title{
EL DESARROLLO DE LA COMPETENCIA LÉXICO-SEMÁNTICA A TRAVÉS DE LA MORFOLOGÍA LÉXICA
}

\author{
Teresa Rodríguez Montes \\ Universidad de Salamanca
}

\section{Resumen}

La morfología léxica puede llegar a ejercer una influencia clave en el desarrollo de la competencia léxico-semántica del aprendiz de español, aunque en muchas ocasiones se presenten como realidades lingüísticas desvinculadas. El conocimiento de los mecanismos morfológicos de la lengua no solo ayuda a la comprensión del significado de términos desconocidos, sino que también incrementa la productividad léxica, mejora el acceso a los términos almacenados en el lexicón mental y permite al hablante manejar con más precisión los significados de los términos conocidos. De este modo, el conocimiento de las reglas de inflexión y derivación morfológicas se convierte en una vía para acceder a un nivel lingüístico diferente y reforzar, desde una doble perspectiva, la competencia comunicativa del hablante. Este hecho obliga a reconsiderar la importancia que se otorga a la morfología léxica tanto en el ámbito de la enseñanza de Español como Lengua Extranjera (E/LE) como en la enseńanza de Lengua Española en la Educación Secundaria.

Palabras Clave: morfología, semántica, léxico, aprendizaje, metodología.

\section{THE DEVELOPMENT OF LEXICAL-SEMANTIC COMPETENCE THROUGH DERIVATIVE MORPHOLOGY}

\begin{abstract}
Derivative morphology can have a key influence on the development of the lexical-semantic competence of the Spanish learner, although these linguistic realities are often presented as unrelated. Knowing the morphological mechanisms of the language not only helps to understand the meaning of unknown terms, but also increases lexical productivity, improves access to the terms stored in the mental lexicon and allows the speaker to handle the meanings of known terms more precisely. In this way, the knowledge of the rules of inflection and morphological derivation becomes a method to access a different linguistic level and reinforce, from a double perspective, the communicative competence of the speaker. This fact makes it necessary to reconsider the importance given to derivative morphology both in the teaching of Spanish as a foreign language and in the teaching of the Spanish Language in Secondary Education.
\end{abstract}

KEYWORDs: morphology, semantics, vocabulary, learning, methodology.

DOI: https://doi.org/10.25145/j.refiull.2020.40.13

Revista de Filología, 40; enero 2020, pp. 275-288; ISSN: e-2530-8548 


\section{INTRODUCCIÓN}

El objetivo principal de todo estudiante de una lengua extranjera es adquirir un dominio del idioma que le permita comunicarse de manera eficaz y superar los obstáculos que puedan surgir durante el intercambio de información. Esto hace que el aprendizaje de una lengua se convierta en un proceso complejo en el que entran en juego una serie de habilidades generales y lingüísticas que deben ser utilizadas de manera adecuada. Por ello, el propósito del profesor debe ser enseñar al aprendiz a utilizar el idioma eficazmente en múltiples contextos. En este ámbito, el de la enseńanza y aprendizaje de una lengua extranjera, la competencia comunicativa se sitúa en el centro de este proceso, ya que es la que posibilita que el intercambio lingüístico se desarrolle con éxito. Esta competencia, formada por una serie de conocimientos, destrezas y habilidades, engloba diversas subcompetencias, entre las que figura la lingüística, el principal foco de interés de este trabajo. A pesar de que todos estos aspectos se interrelacionan de tal modo que cuando uno falla se ve afectado todo el proceso, tiene sentido establecer una serie de compartimentos que permitan profundizar en cada uno de estos ámbitos, aunque las fronteras, en muchos casos, sean prácticamente invisibles.

El Marco Común Europeo de Referencia para las lenguas (MCER) reconoce que la competencia comunicativa engloba tres componentes: el lingüístico, el sociolingüístico y el pragmático. A su vez, considera que el componente lingüístico está integrado por seis subcompetencias: léxica, gramatical, semántica, fonológica, ortográfica y ortoépica, y, aunque es la interacción de todas ellas la que permite que el sistema al que llamamos lengua funcione, no todas han corrido la misma suerte en la historia de la enseñanza de idiomas. La gramática ha ocupado un lugar preferente en las aulas durante décadas y no ha sido hasta hace menos de un siglo cuando a materias como la semántica se les ha reconocido el papel protagonista en este ámbito educativo. Entre todos los componentes lingüísticos a los que la gramática ha hecho sombra durante tantos ańos, el léxico ha sido uno de los más afectados, bien por no contemplarse en los diversos enfoques o bien por utilizar una metodología inadecuada cuando se le prestaba atención. No obstante, durante las últimas décadas, la enseñanza de segundas lenguas ha asistido a una revalorización de este componente, de tal modo que técnicas como la memorización se han dejado a un lado tras comprobar que no capacitaban al estudiante para interiorizar y utilizar de forma adecuada las palabras aprendidas.

En la actualidad el aprendizaje de vocabulario se entiende como una actividad autónoma que requiere de estrategias específicas, puesto que ya no se identifica con una mera memorización de los términos, sino con el desarrollo cualitativo del lexicón mental. Por estas razones, las metodologías actuales suelen abogar por un aprendizaje más consciente del léxico y tratan de integrar este aspecto lingüístico como una parte esencial en la enseñanza de idiomas. Este importante cambio ya se ve reflejado a finales de los años 90 en opiniones como la de Gómez Molina, que reconoce que

la enseñanza del léxico tiene como finalidad que las unidades léxicas pasen a la competencia comunicativa del individuo, dado el valor del vocabulario como 
elemento estructurador del pensamiento -función simbólica- y su necesidad para la interacción social -función comunicativa, tal como manifiesta Allen (1983)-. Es evidente que el léxico es el componente lingüístico que mejor traba el conocimiento del mundo y la competencia comunicativa de un hablante [...] (Gómez Molina 1997: 2).

El hecho de que el dominio léxico sea clave no solo en la competencia lingüística, sino también en la comunicativa, hace que el aprendizaje de vocabulario se convierta en una de las principales metas en el aula de lenguas extranjeras. De este modo, la aplicación de una metodología específica que potencie el desarrollo de la competencia léxico-semántica se vuelve requisito indispensable para convertir al alumno de español en un hablante capaz de comprender y transmitir la información lingüística de forma eficaz.

\section{COMPETENCIA LÉXICO-SEMÁNTICA Y MORFOLOGÍA LÉXICA}

Esta revalorización del componente léxico ha propiciado que los estudios sobre este ámbito lingüístico se multipliquen y que, gracias a ello, sea posible entender mejor cómo se produce el almacenamiento y la recuperación de las palabras y su significado en el lexicón mental del hablante. Tanto en el caso de la lengua materna como en el de segundas lenguas, este proceso parte del almacenamiento y procesamiento de las diferentes unidades, que es lo que posibilita una posterior producción. Durante estas fases el hablante debe adquirir las combinaciones forma-significado y la distribución de las unidades léxicas, pero ¿cómo se desarrolla este proceso en el aula? Existen diversas técnicas que pueden ponerse en práctica, y todas ellas serán válidas siempre que logren que el estudiante interiorice el significado de la palabra, de tal modo que aprenda a usarla correctamente y esté disponible en su lexicón mental cuando la necesite.

Estas propuestas pueden incluirse, en líneas generales, dentro de dos corrientes metodológicas diferentes que, aunque en ocasiones se han considerado como opuestas, no son excluyentes sino complementarias. La primera se correspondería con una enseñanza explícita o directa de vocabulario. Esta metodología, basada en el reconocimiento y análisis de las palabras, busca el procesamiento de las unidades léxicas en profundidad y persigue la interiorización del significado de forma consciente. La segunda posibilidad, vinculada con una enseñanza implícita o indirecta, tomaría como base la extracción de significado de una palabra a partir del contexto en el que aparezca. Desde esta perspectiva se presta una especial atención a la deducción del significado y se busca que la asimilación de los términos se produzca mediante la realización de tareas que no tienen como meta principal el aprendizaje de vocabulario, como la lectura extensiva.

No obstante, a estas dos propuestas se debe añadir una tercera, la que contempla el desarrollo de la competencia léxico-semántica mediante el reconocimiento de los mecanismos morfológicos de la lengua meta. Esta opción considera el cono- 
cimiento morfológico una herramienta indispensable para adquirir la combinación forma-significado, puesto que conocer tanto los afijos como los procedimientos de formación de palabras será de utilidad para comprender el significado de términos desconocidos, incrementará la productividad léxica, mejorará el acceso a los términos almacenados en el lexicón mental y ayudará a conocer no solo la estructura interna de las palabras, sino también su categoría gramatical y las relaciones semánticas y formales que puedan establecer con otros términos. De este modo, la competencia léxica, la semántica y la gramatical encuentran un punto en común en el proceso de aprendizaje con el que se enfrentará el aprendiz del español.

Esta tercera posibilidad está estrechamente vinculada con el desarrollo del componente estratégico, fundamental para sortear las dificultades que puedan surgir durante el proceso comunicativo y compensar así las carencias del hablante. En este sentido, si las estrategias de expresión y de comprensión permiten hacer una aproximación al significado de términos desconocidos, parece lógico contemplar la morfología léxica de la lengua meta como una vía para desarrollar este componente. Según el MCER, entre las estrategias de expresión se encuentran las de evitación y las de aprovechamiento, ambas de tipo compensatorio y muy utilizadas cuando se está aprendiendo un idioma. Así, cuando el estudiante no tiene almacenado en su lexicón mental el término que está buscando tratará de sustituirlo o explicarlo haciendo uso de mecanismos como el circunloquio. Del mismo modo, también puede usar de forma estratégica sus conocimientos morfológicos para formar una palabra derivada que exprese la idea que tiene en mente, siempre y cuando las reglas del idioma lo permitan.

Si se enfoca la enseñanza de esta parcela lingüística desde esta óptica, no cabe duda de que esta permitirá al hablante mejorar su conocimiento interno de la lengua y, por tanto, emplear el idioma con mayor soltura. Asimismo, el aprendiz encontrará en la lexicogénesis el perfecto aliado para desarrollar estrategias de comprensión, expresión y deducción; al fin y al cabo, tener un buen dominio del vocabulario no dependerá únicamente del número de palabras conocidas, sino también de las capacidades que permitan acceder al significado de términos desconocidos. De esta forma, el hablante podrá ampliar su caudal léxico mediante la incorporación de nuevos términos al lexicón mental a través del conocimiento de las reglas y procedimientos de derivación de la lengua meta.

Estos supuestos se ven reforzados por diversos estudios que demuestran que las palabras con morfología se adquieren más fácilmente que las que carecen de ella, porque en el lexicón mental estos afijos no se almacenan junto a las raíces, sino de manera independiente, lo que permite reconocerlos en palabras nuevas o a ańadirlos a una raíz diferente. En el estudio realizado por Hernández Muñoz (2015) ${ }^{1}$ se vin-

${ }^{1}$ Los resultados de esta investigación fueron publicados en el artículo «¿Es el conocimiento morfológico un mecanismo determinante en la recuperación del léxico disponible?». Este estudio, aunque elaborado desde la perspectiva de la competencia léxica en la lengua materna y centrado en el ámbito temático de las profesiones, tiene vigencia y aplicabilidad también para los aprendices de español. 
cula el conocimiento morfológico con la recuperación del léxico disponible y se establece que es posible relacionar la capacidad de producción de un número mayor de palabras asociadas a un tema concreto con estrategias lingüísticas internas como la habilidad para crear palabras nuevas, activar un mayor número de sufijos o ampliar la productividad de la base léxica. Este hecho demuestra que tener un control razonable de las estructuras morfológicas de la lengua permite una mejor estructuración del lexicón mental, ya que, tal y como reconoce la autora:

Cuanto mayor sea el conocimiento metalingüístico de los mecanismos internos de la formación de palabras, mejores son las capacidades de almacenamiento y recuperación de las palabras desde la memoria semántica. En general, el conocimiento metalingüístico morfológico es uno de los motores más importantes para mejorar el dominio general del sistema lingüístico (Hernández Muñoz 2015: 260).

Los planteamientos expuestos hasta ahora ponen de manifiesto la importancia de la didáctica de la morfología léxica; no obstante, la atención que recibe esta parcela en el ámbito de E/LE suele ser mínima. Como reconoce Campillejo García (2015), apenas encontramos estudios o propuestas que profundicen en cómo se pueden transmitir a los discentes los mecanismos de composición y derivación de tal forma que les sean útiles para comprender y crear palabras de manera autónoma, correcta y comunicativamente pertinente.

\section{LA DIDÁCTICA DE LA MORFOLOGÍA LÉXICA EN EL AULA DE E/LE}

Este vacío de información y propuestas sobre la lexicogénesis en el ámbito de $\mathrm{E} / \mathrm{LE}$ ha despertado el interés de aquellos que se preguntan por qué a estos contenidos aún no se les ha otorgado el valor que realmente merecen. De hecho, una revisión de los diferentes manuales utilizados en este contexto educativo pone de relieve que la morfología léxica suele quedar relegada a un segundo plano. Esta realidad se ve reflejada en las reflexiones de profesionales como Serrano-Dolader, que afirma:

La didáctica de la lengua de corte tradicional ha reflexionado con frecuencia sobre los mecanismos de formación de palabras, si bien es cierto que normalmente lo ha hecho con poca fortuna en sus posibilidades reales de una aplicación fructífera en el aula de ELE/EL2. Los enfoques comunicativos en la enseñanza-aprendizaje de idiomas, por su parte, no han dedicado a los procesos lexicogenéticos la atención que merecen. El manifiesto interés ofrecido por estos enfoques en relación con el vocabulario de la lengua meta no ha venido acompañado de una reflexión pausada sobre el papel desempeñado por la morfología léxica en el proceso de progresivo dominio de la lengua (Serrano-Dolader 2018: 13).

Parece, por tanto, que la última responsabilidad recae en el profesor de E/LE, pues si elige introducir estas cuestiones en el aula debe considerar de qué forma, en qué momento hacerlo y cómo sortear los obstáculos que puede plantear 
trabajar con la morfología. Para ello debe, en primer lugar, valorar la presencia de esta disciplina en el ámbito de E/LE de forma objetiva. Por esta razón es indispensable que el primer paso sea acudir a dos documentos normativos que sirven como base para la confección de los diversos materiales en este ámbito educativo: el Marco Común Europeo de referencia (MCER) y el Plan Curricular del Instituto Cervantes (PCIC). Aunque la finalidad de estos documentos no es proporcionar aplicaciones prácticas, sino servir como guía en el proceso de enseñanza de español con el fin de lograr cierta homogeneidad, es importante valorar qué directrices establecen con respecto a la enseñanza del aspecto lingüístico que nos ocupa.

El MCER considera la morfología como uno de los subcomponentes básicos de la competencia gramatical. Con respecto a la morfología léxica en concreto, se establece que, entre las diversas formas de desarrollarla, puede incluirse la presentación de paradigmas formales y tablas a los que precederán una serie de explicaciones en las que se utilizará un metalenguaje apropiado y diversos ejercicios. No obstante, no se proporciona ninguna escala que ayude a medir el grado de dominio de esta subcompetencia ni se establecen criterios que ayuden a determinar qué debe saber el aprendiz según el nivel en el que se encuentre. Sí se ofrecen unas nociones teóricas básicas sobre esta disciplina, pero carecen de utilidad real para ser implementadas en el aula mediante el desarrollo de propuestas prácticas:

La morfología se ocupa de la organización interna de las palabras. Las palabras se pueden analizar como morfemas, clasificados de la siguiente forma: raíces, afijos (prefijos, sufijos, infijos), que comprenden afijos de derivación y afijos de flexión. Las palabras se pueden clasificar en palabras simples, palabras complejas (raíz + afijos), palabras compuestas y lexías complejas (que contienen más de una palabra). La morfología también se ocupa de otras maneras de modificar formas de palabras; por ejemplo: alternancia de vocales, modificación de las consonantes, formas irregulares, flexión y formas invariables (MCER 2002: 111-112).

Finalmente, se considera que «los usuarios del Marco de referencia pueden tener presente y, en su caso, determinar qué elementos morfológicos y procesos tendrá que dominar el alumno, o cómo se le capacitará para ello, o qué se le exigirá al respecto». Pero, además de ello, el profesor debe ingeniárselas para plantear estas cuestiones desde una perspectiva útil, puesto que en este documento no se contemplan los conocimientos morfológicos ni como un mecanismo que facilite la aproximación a significados desconocidos o mejore la competencia léxico-semántica ni como una vía para desarrollar el componente estratégico. Una vez realizada esta consulta, el usuario debe acudir al PCIC, que supone la concreción de las directrices incluidas en el MCER para la enseñanza de español. En este documento aparecen también algunas pinceladas sobre este subcomponente lingüístico, aunque tampoco se le presta la atención necesaria ni se trata desde una perspectiva del todo idónea.

En la introducción que precede al apartado que trata la gramática, se especifica que no se incluye ninguna sección referida a la morfología léxica o composicional porque determinar qué prefijos, sufijos o procedimientos deben incluirse en cada nivel sería una tarea casi imposible. Por otra parte, se expone que esta cuestión 
es más de tipo léxico y metodológico que de tipo gramatical y que, por ello, necesita ser tratada desde una perspectiva pedagógica que va más allá de la mera definición de contenidos. A pesar de ello, en algunos apartados figuran algunos afijos presentados de forma esquemática; la presencia de los prefijos y sufijos aumenta conforme lo hace el nivel. En la mayoría de los casos, se trata de diminutivos y aumentativos que se presentan como recursos gramaticales para intensificar los elementos del discurso y para expresar el grado superlativo, tal y como se observa en la siguiente tabla:

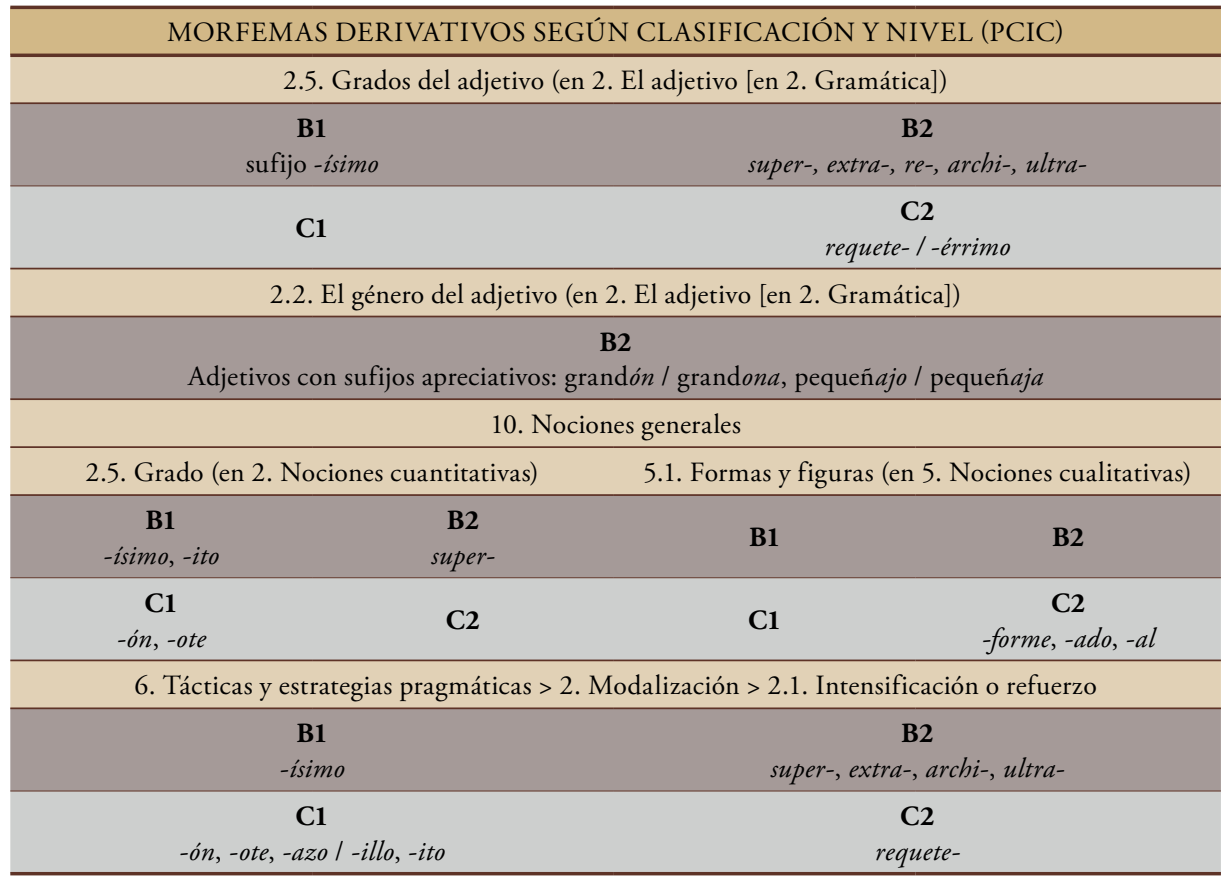

La información recopilada demuestra que la morfología léxica apreciativa tiene un peso mucho mayor que la nocional, que aparece en ocasiones puntuales. Los afijos de este tipo que figuran son, por ejemplo, los sufijos -forme, -ado y -al. Los tres se presentan como mecanismos para designar una forma tal y como muestran los ejemplos (oviforme, acampanado, piramidal), pero los dos últimos pueden añadir a una palabra compuesta muchos otros significados que no se incluyen en ningún apartado. El único afijo al que se presta una mayor atención es el sufijo -mente, sobre el que se introducen diversas informaciones en el apartado de gramática que se desarrollan en los diversos niveles. Por ejemplo, en el nivel B2 se hace referencia a las restricciones de estos adverbios a partir de los adjetivos deverbales terminados en -ble, -do y -nte, pero estos afijos no se mencionan en ningún otro apartado. Este análisis demuestra que las referencias a la morfología significativa no aparecen integradas de forma progresiva y que no hay una selección de afijos que parta de unos criterios determinados. 
Las directrices que se incluyen en estos dos documentos -y otros contenidos que no figuran en ellos- deberían materializarse en los manuales y materiales utilizados en las clases de E/LE, que supondrían un nivel de concreción mayor al servir como enlace entre los supuestos teóricos y un contexto de enseñanza-aprendizaje real. Una revisión de diversos manuales permitió establecer la conclusión de que, una vez más, se suele prestar más atención a la morfología flexiva y a la derivación apreciativa, mientras que las cuestiones relacionadas con la formación de palabras tienden a ser presentadas a partir de tablas o figuras que no constituyen el foco principal del aprendizaje. En cuanto a la práctica, las actividades propuestas no tienden a promover un uso reflexivo de la lengua ya que consisten en una aplicación mecánica de conocimientos. Tal y como reconoce Campillejo García:

Cuando se incluyen cuestiones relativas a cualquiera de los procesos de morfología léxica, estas suelen aparecer como refuerzo de las explicaciones de otros aspectos gramaticales, tal es el caso del género y el número de los sustantivos o los adjetivos. En otras ocasiones, se insertan algunos ejemplos de compuestos o derivados en recuadros colocados en el margen de las páginas bajo el título ¿¿Sabes? o Recuerda, como si se tratara de algo adicional y de poca importancia (Campillejo García 2015: 23).

Esta falta de propuestas hace que el profesor de E/LE tenga dificultades al confeccionar materiales que integren la enseñanza de la lexicogénesis; no obstante, cada vez son más los profesionales que tratan de proponer ideas y directrices que allanen este camino. Lo que aquí se defiende es que el éxito del proceso reside en lograr que el aprendiz active una serie de mecanismos que le permitan entender el estudio de ciertas cuestiones morfológicas como una vía para acceder al componente léxico, de tal manera que la interiorización de la combinación forma-significado no sea una realidad nueva y ajena a él, sino una realidad sobre la que también se puede reflexionar. Es decir, se persigue que el estudiante desarrolle una conciencia morfológica ${ }^{2}$ que le permita incorporar los conocimientos y habilidades adquiridos a su realidad lingüística. Pero ¿cómo consigue esto el profesor de E/LE?

En primer lugar, según advierte Serrano-Dolader (2018), pueden pautarse metodológicamente tres fases para que el proceso de adquisición de la morfología léxica se desarrolle de forma adecuada: primero se debe reconocer la forma con el fin de identificar y analizar los constituyentes, seguidamente se les asignará un significado y, finalmente, se producirán las nuevas palabras complejas. Este proceso, que se refuerza con la práctica, tiene en cuenta la capacidad autónoma del aprendiente y puede servir como elemento motivador ya que él mismo se convierte en protagonista de su propio aprendizaje al predecir significados, descubrir combinaciones y generar nuevas formas. No obstante, si la palabra que produce no es correcta, no

${ }^{2}$ La conciencia morfológica puede definirse como «el aspecto de la conciencia metalingüística que se refiere al plano consciente de las estructuras morfémicas de las palabras y su habilidad para la reflexión y manipulación de dichas estructuras» (González, Rodríguez y Gázquez, 2011: 142). 
debe sentirse frustrado ni enfocar su producción como un error, puesto que puede ser un síntoma de que el sistema se está interiorizando. De hecho, quizá su creación no esté aceptada normativamente, pero es probable que pueda captarse su significado con facilidad (ej.: terrizar por aterrizar).

En segundo lugar, es esencial determinar cómo integrar estas cuestiones en el proceso de enseñanza-aprendizaje, pues puede hacerse desde diferentes ópticas: a) desde una perspectiva formal en la que se trabaje con palabras de diferentes categorías, b) tomando como punto de partida un afijo determinado para explorar las posibles combinaciones, c) utilizando un ámbito temático concreto y combinando diferentes afijos y diferentes categorías formales o d) relacionando la morfología con otros niveles de la lengua, como el sintáctico. Finalmente, es importante no perder de vista tres puntos clave: a) presentar la morfología léxica como un proceso de enriquecimiento léxico ya que posibilita la formación y el entendimiento de nuevas palabras, b) apelar a la capacidad de deducción del estudiante y promover que utilice sus conocimientos previos para desarrollar y crear nuevas estrategias y c) valorar si los contenidos y las actividades permitirán que el aprendiz comprenda el vocabulario a la par que desarrolla estrategias de aprendizaje y desarrolla su capacidad de deducción.

Del mismo modo, es de capital importancia que el profesor tenga en cuenta durante todo el proceso que no toda la morfología léxica del español es igualmente útil para el alumno de E/LE ni puede trasladarse al aula con la misma eficacia. Por ello, se debe valorar el grado de operatividad de las nociones que se pretenden trabajar; cuestiones como la frecuencia de uso, la productividad, la transparencia del derivado y su grado de composicionalidad deben ser tenidas en cuenta para la selección de contenidos y posterior confección de materiales. Asimismo, hay que considerar el posible contraste que pueda existir con los procesos lexicogenéticos de la lengua materna del aprendiente, por lo que estas cuestiones no se trabajarán igual si la clase es de hablantes monolingües o si nuestros aprendices tienen lenguas maternas que difieren en estos procesos. Valorar la influencia de todos estos factores es clave para que el proceso de aprendizaje se realice con éxito y las nociones interiorizadas se conviertan en una herramienta que facilite la comprensión y expresión del idioma.

\section{LA INTEGRACIÓN DE CONTENIDOS: HACIA UNA PROPUESTA DIDÁCTICA}

Una vez establecidos los supuestos teóricos que deben tenerse en cuenta para trabajar con la morfología léxica en el aula de E/LE, el profesor debe seleccionar una serie de contenidos, adaptarlos a su realidad educativa y ponerlos en práctica para que se efectúe un aprendizaje significativo. Cada situación particular dará lugar a una propuesta didáctica diferente, que aumentará su validez si su principal fin es que el aprendiz desarrolle una conciencia morfológica a través de la reflexión lingüística y active sus conocimientos previos, sus habilidades lingüísticas y su capacidad de deducción. Por ello, es básico facilitarle las herramientas que le permitan iniciar el proceso de adquisición de la morfología léxica para que, final- 
mente, estos mecanismos se pongan en funcionamiento de manera automática al entrar en contacto con la lengua meta. Con el fin de aportar un apoyo que pueda servir al docente para trabajar con estos contenidos en el aula, se presentará el proceso de selección de contenidos y nociones realizado en la confección de una propuesta diseñada para el aula de E/LE.

Para diseñar cualquier unidad didáctica, primero debe hacerse una selección de la información que se va a incluir. Uno de los principales problemas que plantea la introducción de estas nociones en el aula es, tal y como reconoce el PCIC, determinar qué afijos incluir en cada nivel. Esta dificultad de distribuir los morfemas derivativos por niveles se debe a varios factores: por una parte, cuestiones intrínsecas del morfema tales como su productividad o su significado; por otra parte, existen también dificultades referentes a la base léxica con las que se trabaje, como los cambios en la categoría o en la raíz del término. Por estas razones, debe prestarse atención a diversos factores y valorar tanto los términos que se quieren enseñar y los morfemas con los que pueden funcionar como la forma, significado y frecuencia de uso de la palabra que se extrae de su combinación. Asimismo, debe contemplarse también la introducción de otras cuestiones lingüísticas que permitan al aprendiz poner en relación los diferentes componentes que entran en juego en la comunicación.

La propuesta referida toma como punto de partida las posibles equivalencias y diferencias entre un sintagma preposicional encabezado por la preposición de y algunos adjetivos formados por derivación (como sucede, por ejemplo, en de metal frente a metálico), ambos utilizados como complemento del nombre. Como ámbito temático, se eligió el de las materias y materiales, puesto que su inclusión en el PCIC podría servir para determinar, de forma aproximada, en qué nivel introducir los diferentes términos o expresiones. De este modo, en la propuesta se agrupan contenidos sintácticos (sintagma preposicional formado por $d e+$ 'material'), funcionales (uso de estos sintagmas como complemento del nombre), morfológicos (equivalencias y diferencias con posibles adjetivos derivados del término que conforma el sintagma), léxicos (qué términos se obtienen al añadir un determinado afijo) y semánticos (qué significado adquiere el adjetivo en función del afijo aplicado). El hecho de partir de un ámbito temático y de una estructura tan específicos no implica que toda la propuesta deba estructurarse en torno a estas ideas ya que, por ejemplo, se utilizó también para trabajar los gentilicios, pues la estructura de + 'lugar de origen' siempre es equivalente a este tipo de adjetivos.

Aprender estas equivalencias será de gran utilidad especialmente en los niveles superiores, en los que manejar un vocabulario más específico permitirá al aprendiz construir un discurso más preciso. Además, en ocasiones un sintagma preposicional no soluciona el problema comunicativo, pues para decir que una comida tiene mucha agua, no se puede utilizar el sintagma comida de agua, y el hablante demostrará, sin lugar a duda, que su repertorio léxico es más amplio si dice que su comida está aguada. Algo parecido sucede cuando se interpreta el sintagma preposicional de oro como equivalente del adjetivo dorado. Por tanto, una vez que el estudiante interioriza el término oro y su significado, es útil que sepa que, si le dan a elegir entre un reloj dorado y un reloj de oro, saldrá más beneficiado si escoge el último. 
Para hacer una selección de los términos a partir de los que trabajar la morfología, se acudió al apartado del PCIC denominado "materia», incluido en la categoría «Nociones generales». Entre los términos relevantes figuraban los siguientes: en el nivel A2 madera, piel, plástico, metal, tela, papel, cristal; en el nivel B1 acero, aluminio, hierro, oro, plata, piedra, cuero, cartón; en el nivel B2 bronce, cobre, plomo; mármol, cemento, ladrillo; cerámica, barro; cera, hueso, plumas; metálico, textil; y, finalmente, en el nivel C1 platino, diamante; corcho, goma, caucho; granito, gres, yeso, escayola, cartón piedra. Una vez realizada esta selección, se confeccionó una tabla con este listado de materiales y a partir de ellos se formaron los diversos derivados que existen en nuestro idioma. Los morfemas derivativos seleccionados fueron los siguientes: - eo (sufijo utilizado con bases cultas como ocurre en aéreo, férreo o áureo), -ico (sufijo utilizado para formar términos como metálico, cerámico o acuático), -oso (sufijo utilizado en acuoso, vidrioso o rocoso), -ado (sufijo presente en dorado, plateado o aguado), y otros sufijos de menor productividad como -eño, -izo o -ino (utilizados para formar adjetivos como marfileño, plomizo o cristalino).

Se escogieron también algunas formas y expresiones verbales que permiten referirse al material con el que está hecho algo con el fin de trabajar con mecanismos como la paráfrasis y valorar si podían ser sustituidas por términos derivados. Estas expresiones fueron ser de + material (nivel A2), estar hecho de + material (nivel B1) y estar fabricado con + material (nivel B2). Estas estructuras pueden funcionar también como complemento del nombre: de + material, hecho de + material, fabricado con + material y, aunque no están directamente relacionadas con la morfología léxica, se mantuvieron porque ofrecen la posibilidad de parafrasear determinados adjetivos y de explicar características concretas para las que no existe un adjetivo o un verbo determinado. La selección de términos y expresiones se dividió según diferentes niveles en función de la base léxica utilizada y el afijo añadido, de forma que en la propuesta realizada se tuvieron en cuenta dos niveles diferentes: B2 y C1.

Los morfemas derivativos con los que se trabajaba en el nivel B2 eran -ico, -oso y -ado porque, además de tener una alta productividad, permitían trabajar y comparar el significado de diferentes términos derivados que se usan con mayor frecuencia. Por otra parte, los sustantivos seleccionados fueron plástico, metal, tela, papel, cristal, oro, plata, bronce y cristal. Esta selección hizo posible comparar las diferencias que aporta un sufijo en función de la base léxica (por ejemplo, dorado como sinónimo de la expresión que parece de oro y aguado como sinónimo de la expresión que contiene demasiada agua) y trabajar también con un mismo término y los matices de significado que puede aportarle cada sufijo (tal y como sucede con aguado, acuoso y acuático). Para la propuesta destinada a niveles más altos se trabajó con el sufijo - eo y con los que tenían una productividad más baja en este ámbito: -eño, -izo e -ino. La inclusión del sufijo - eo en este apartado se debe a una cuestión formal, ya que las bases léxicas deben utilizar una raíz diferente para formar los términos derivados (áureo, férreo, ígneo, vítreo, óseo, marmóreo, ebúrneo, broncíneo, nacáreo, céreo y térreo). En este caso se buscaba desarrollar la precisión léxica y trabajar con un nivel de lengua más culto, por lo que los ejemplos utilizados en la unidad fueron extraídos de varios corpus lingüísticos, con el fin de que el aprendiz se familiarizara con el tipo de registro en el que suelen utilizarse estos términos. 
Con respecto a los elementos extralingüísticos, cabe destacar como componente esencial la motivación, pues es clave convencer al aprendiz de que este tipo de actividades le ayudarán a incrementar sus habilidades léxico-semánticas al desarrollar nuevas estrategias de deducción de significado, parafrasear términos y crear nuevas palabras. A su vez, debe estructurarse en torno a un enfoque comunicativo y cooperativo que permita aprender el léxico de forma contextualizada y planificada. Finalmente, el profesor tiene que valorar la utilidad y las posibilidades de aprovechamiento de las nociones presentadas y hacerlo mediante una propuesta que combine teoría y práctica desde una perspectiva dinámica, activa y motivadora que ayude al alumno a descubrir el funcionamiento de la lengua que está aprendiendo.

\section{APLICACIONES MÁS ALLÁ DEL AULA DE E/LE. CONCLUSIONES}

A lo largo de estas páginas se ha hecho referencia a la influencia que la morfología léxica puede llegar a ejercer en los hablantes de E/LE. No obstante, también es posible trasladar estos supuestos a la realidad de la asignatura Lengua Castellana y Literatura en las aulas de Educación Secundaria, donde la enseñanza de este subcomponente lingüístico ya no se contempla como una opción, sino como algo obligatorio. Mientras que en el aula de E/LE la inclusión de la morfología léxica es una opción para potenciar el desarrollo de la competencia léxico-semántica, en este otro ámbito educativo es un contenido obligatorio que, en la mayor parte de los casos, el estudiante siente como un conocimiento que carece de una aplicación real. Esto hace que el estudiante de Secundaria entienda la morfología como una disciplina abstracta carente de utilidad y cuyo aprendizaje no le proporcionará ningún beneficio en su futuro más próximo. Las principales causas de este sentimiento son la total desconexión entre los contenidos enseñados y la realidad del alumno, así como un aislamiento de la disciplina de su vertiente funcional. Todo ello desemboca en una falta de motivación y de interés en las aulas de Lengua, lo que tiene, como última consecuencia, la formación de hablantes con escasa competencia comunicativa.

Por esta razón, aprovechar las posibilidades que brinda la morfología léxica es determinante también en el aula de Secundaria, aún más si tenemos en cuenta que apenas se presta atención al aprendizaje de vocabulario en esta etapa educativa. Quizá la principal explicación sea que, en ocasiones, se considera que la intuición que un hablante nativo tiene de su propia lengua es suficiente para identificar los lexemas que forman un término derivado, saber a qué categoría gramatical pertenece o qué relaciones puede establecer con otros términos. No obstante, no siempre el estudiante de Secundaria dispone de mecanismos para extraer esta información. Asimismo, si se tiene en cuenta que una de las principales dificultades para comprender un texto con las que pueden encontrarse los adolescentes es la aparición de términos desconocidos, parece lógico que el profesor de Secundaria también proporcione a sus estudiantes herramientas que les permitan solventar estos obstáculos. Por otra parte, tampoco se debe olvidar que el desarrollo de las habilidades lingüísticas relacionadas con el aprendizaje de nuevas unidades léxicas y significados 
de otras ya aprendidas nunca se detiene, por lo que el hablante nativo de español también encontrará útil mejorar su competencia léxico-semántica. De este modo, ayudarlo a ampliar y mejorar sus habilidades para que disponga de recursos léxicos variados y aprenda a utilizarlos con precisión es también de capital importancia.

Por todas las razones expuestas, cada vez son más los docentes que consideran que integrar los aspectos teórico-prácticos que guardan relación con la formación de palabras es algo esencial en la enseñanza del idioma, independientemente de que se haga desde la perspectiva de L1 o de L2, pues no solo posibilita un mejor acceso al vocabulario, sino que permite manejarlo con mayor precisión y desarrollar estrategias de deducción, de comprensión y de expresión. Es cierto que una desacertada presentación de estas nociones lingüísticas puede abrumar al estudiante y generar un efecto contraproducente en su aprendizaje, así que es importante recordar que no se trata de convertir al alumno en un experto en morfología, sino de que aprenda a emplear estos conocimientos de manera estratégica y sepa utilizar de forma práctica estos aspectos teóricos. Por estas razones, el objetivo principal debe ser concienciarlo de que interiorizar cómo funcionan determinados afijos es algo verdaderamente útil tanto para reconocer el significado y la categoría gramatical de las palabras como para ampliar su vocabulario. $\mathrm{Y}$, aunque la complejidad y abstracción de la disciplina hagan que integrar de forma correcta estas nociones en el aula sea un reto, si el profesor decide aceptarlo no solo estará formando mejores hablantes de español, sino que también se estará dando a sí mismo la oportunidad de convertirse en un profesional más competente.

ReCibido: mayo de 2019; ACEPTADo: octubre de 2019. 


\section{BIBLIOGRAFÍA}

Allen, Virginia French (1983): Techniques in Teaching Vocabulary, Oxford: Oxford University Press.

Baralo Ottonello, Marta (1996): «Algunos aspectos de la adquisición de la morfología léxica del español como lengua extranjera", Actas del V Congreso Internacional de ASELE: 143-150.

Campillejo García, Rosa (2015): La formación de palabras en los manuales de E/LE. Memoria de máster inédita, Cáceres: Universidad de Extremadura.

Castillo Carballo, María Auxiliadora, Juan Manuel García Platero y Juan Pablo Mora GutiéRREZ (2003): «¿Cómo enseñar lexicogénesis a hablantes de otras lenguas?», Actas del XIII Congreso Internacional de ASELE: 880-889.

Cervera Mata, Teresa (2012): «El léxico y la enseñanza de la lengua: innovación y propuestas didácticas a partir del análisis de un método de enseñanza de español», Enunciación 17 (2): 138-154.

Consejo de Europa (2002): Marco común europeo de referencia para las lenguas: aprendizaje, enseñanza, evaluación. URL: https://cvc.cervantes.es/ensenanza/biblioteca_ele/marco/cvc_ mer.pdf; 08/05/2019.

Díaz Hormigo, María Tadea (2011): «Formación de palabras y ELE: una propuesta didáctica para la enseñanza de la formación de verbos por prefijación en español a discentes italianos», Revista de Didáctica Español Lengua Extranjera 22.

García Jerez, Ana María (2006): Procesos de formación de palabras: la derivación en la enseñanza del español como lengua extranjera. URL: http://www.educacionyfp.gob.es/educacion/mc/ redele/biblioteca-virtual/numerosanteriores/2006/memoriamaster/2-semestre/garcia-j. html; 08/05/2019.

Gómez Molina, José Ramón (1997): «El léxico y su didáctica: una propuesta metodológica», REALE: revista de estudios de adquisición de la lengua española 7: 69-94.

González Sánchez, Lorena, Celestino Rodríguez Pérez, José Jesús Gázquez Linares (2011): "Aproximación al concepto de conciencia morfológica», Magister: Revista miscelánea de investigación 24: 135-146.

Hernández Muñoz, Natividad (2015): “¿Es el conocimiento morfológico un mecanismo determinante en la recuperación del léxico disponible?», Lenguas, lenguaje y lingüistica. Contribuciones desde la Lingüistica General: 259-268.

Instituto Cervantes (2006): Plan curricular del Instituto Cervantes. Niveles de referencia para el español, Madrid: Instituto Cervantes-Edelsa. URL: http://cvc.cervantes.es/ensenanza/ biblioteca_ele/plan_curricular/; 07/05/2019.

La Torre Ródenas, María Dolores (2000): «La enseñanza de la formación de palabras en la clase de E/LE», Actas del X Congreso Internacional de ASELE: 1047-1056.

Serrano-Dolader, David (2018): Formación de palabras y enseñanza del español LE/L2, London / New York: Routledge.

Serrano-Dolader, David, María Antonia Martín Zorraquino y José Francisco Val Álvaro (2009): Morfología y español como lengua extranjera (E/LE), Zaragoza: Prensas Universitarias de Zaragoza.

Urdampilleta Araolaza, Maider (2017): La formación de palabras en los manuales de E/LE. Trabajo de fin de grado inédito. URL: https://addi.ehu.es/bitstream/handle/10810/23602/ TFG_Urdampilleta.pdf?sequence=1\&isAllowed=y; 01/02/2019.

VARELA Ortega, Soledad (2003): «Léxico, morfología y gramática en la enseñanza de español como lengua extranjera», Estudios de Lingüistica. Universidad de Alicante (ELUA) 17: 571-588. 